Documentation et bibliothèques

DOCUMENTATION BIBLIOTHEQUES

\title{
Dix ans de coopération entre les bibliothèques universitaires du Québec : un bilan
}

\section{Onil Dupuis}

Volume 23, numéro 3, septembre 1977

URI : https://id.erudit.org/iderudit/1055227ar

DOI : https://doi.org/10.7202/1055227ar

Aller au sommaire du numéro

\section{Éditeur(s)}

Association pour l'avancement des sciences et des techniques de la documentation (ASTED)

\section{ISSN}

0315-2340 (imprimé)

2291-8949 (numérique)

Découvrir la revue

\section{Citer cet article}

Dupuis, O. (1977). Dix ans de coopération entre les bibliothèques universitaires du Québec : un bilan. Documentation et bibliothèques, 23(3), 143-150.

https://doi.org/10.7202/1055227ar

\section{Résumé de l'article}

L’année 1977 marque le dixième anniversaire de la création du Sous-comité des bibliothèques de la Conférence des recteurs et des principaux des universités du Québec. Celui-ci a joué, dans les milieux universitaires et même au sein de la collectivité des bibliothèques québécoises, un rôle unique. Il a été l'un des premiers organismes de coopération volontaire à amorcer, et surtout à planifier, une mise en commun de ses ressources au bénéfice de l'ensemble de ses membres et de leurs usagers. L'auteur dresse un rapide bilan des activités du Sous-comité et esquisse quelques-uns de ses projets, après avoir situé, dans le contexte plus large de l'ensemble des activités de coopération des universités elles-mêmes, celles dont le Sous-comité des bibliothèques a été l’instigateur.
Tous droits réservés (C) Association pour l'avancement des sciences et des techniques de la documentation (ASTED), 1977
Ce document est protégé par la loi sur le droit d'auteur. L'utilisation des services d'Érudit (y compris la reproduction) est assujettie à sa politique d'utilisation que vous pouvez consulter en ligne.

https://apropos.erudit.org/fr/usagers/politique-dutilisation/ 


\title{
Dix ans de coopération entre les bibliothèques universitaires du Québec: un bilan
}

\author{
Onil Dupuis \\ Chargé de recherche \\ Conférence des recteurs et des principaux \\ des universités du Québec \\ Montréal
}

L'année 1977 marque le dixième anniversaire de la création du Sous-comité des bibliothèques de la Conférence des recteurs et des principaux des universités du Québec. Celui-ci a joué, dans les milieux universitaires et même au sein de la collectivité des bibliothèques québécoises, un rôle unique. II a été l'un des premiers organismes de coopération volontaire à amorcer, et surtout à planifier, une mise en commun de ses ressources au bénéfice de l'ensemble de ses membres et de leurs usagers. L'auteur dresse un rapide bilan des activités du Sous-comité et esquisse quelques-uns de ses projets, après avoir situé, dans le contexte plus large de l'ensemble des activités de coopération des universités elles-mêmes, celles dont le Sous-comité des bibliothèques a été l'instigateur.

The year 1977 marks the tenth anniversary of the creation of the Sous-comité:des bibliothèques de la Conférence des recteurs et des principaux des universités du Québec. This committee has played a unique role in the university field and even in that of the whole of libraries in Québec. It was one of the first organizations of voluntary cooperation to start and especially to plan a pooling of resources in order to benefit the whole of its membership and their users. The author, after having situated within the larger context of the cooperative efforts of universities themselves, those that the Sous-comité des bibliothèques has initiated, draws a balance sheet of the activities of this committee and describes some of their projects.

El año 1977 marca el décimo aniversario de la créación del subcomité de las bibliotecas de la Conferencia de los rectores y principales de las universidades de Québec. Este ha desempeñado un papel único tanto en el medio universitario como en la colectividad de las bibliotecas quebequenses. Fue una de las primeras organizaciones de cooperación voluntaria en empezar a planificar una utilización comun de los recursos en provecho de sus miembros y usuarios. El autor establece el balance de las actividades del subcomité y expone algunos de sus proyectos, situando en el contexto más amplio de la cooperación universitaria las actividades instauradas por el subcomité de las bibliotecas.

\section{La coopération universitaire}

\section{Rôle et objectifs}

Le ministère de l'Éducation, par sa direction de l'enseignement supérieur, le Conseil des universités, le Conseil supérieur de l'éducation, par sa Commission de l'enseignement supérieur, constituent l'appareil légal et administratif dont s'est doté le gouvernement du Québec dans le domaine de l'enseignement supérieur. Chacun de ces organismes tente d'exercer le rôle et d'assumer les responsabilités que lui définissent 
les lois existantes. Ces structures ne font cependant aucune place à un regroupement, sur le plan institutionnel, des responsables immédiats des universités québécoises.

Or, les recteurs, particulièrement responsables de l'orientation et du dynamisme de la vie universitaire dans leurs institutions respectives, doivent, comme groupe, jouer un rôle d'entraînement qui soit efficace et reconnu par les autorités gouvernementales, le Conseil des universités, la communauté universitaire et la société québécoise.

La Conférence des recteurs et des principaux des universités du Québec (CREPUQ), qui regroupe les dirigeants des universités du Québec, institutions à vocation publique, est un organisme d'intérêt public qui doit jouer un rôle de premier plan auprès des pouvoirs publics en faisant valoir, d'une part les conditions essentielles qui doivent présider à l'orientation et au développement du système d'enseignement supérieur au Québec, d'autre part les conditions requises pour l'exercice et l'épanouissement des fonctions propres aux universités.

Vis-à-vis de ses membres, la Conférence des recteurs se reconnaît une double fonction: elle agit comme organisme de consultation et de coopération inter-institutionnelles et comme organisme d'étude et de réflexion.

À cet effet, elle doit:

a) développer entre ses membres ce climat de.confiance et de solidarité qui permettra de constituer un droit commun sur le plan de l'élaboration et de la mise en œuvre de politiques qui les touchent;

b) promouvoir et favoriser la collaboration des institutions entre elles et la poursuite de projets communs qui permettent une utilisation optimale des ressources;

c) préserver l'autonomie collective des institutions universitaires en fa- vorisant l'élaboration de politiques communes;

d) prendre les moyens nécessaires pour que la communauté universitaire reconnaisse la Conférence des recteurs comme porte-parole autorisé;

e) effectuer les travaux d'analyse et de prévision portant sur toute question que les universités conviendront entre elles de lui confier. ${ }^{1}$

\section{La CREPUQ et le Sous-Comité des bibliothèques}

C'est en mai 1967 que fut juridiquement constituée la CREPUQ selon la troisième partie de la Loi des compagnies, dans le but de favoriser l'échange, l'analyse et la diffusion de renseignements utiles dans la poursuite de ses objectifs ${ }^{2}$. En outre, le 1er janvier 1968, elle retenait les services d'un directeur administratif responsable de la mise sur pied d'un secrétariat permanent et du fonctionnement de la Conférence.

Afin d'atteindre ces divers objectifs, la CREPUQ a établi plusieurs comités et souscomités permanents, dont I'un des plus importants, tant sur le plan de son activité que sur le plan de son rôle par rapport aux activités d'enseignement et de recherche, fut le Comité de coordination des bibliothèques $^{3}$. Ce comité a pour mandat de soumettre des recommandations favorisant un développement planifié et une meilleure

1. Cette définition du rôle et des objectifs de la Conférence a fait l'objet d'une adoption formelle par son Conseil d'administration lors de sa $78 \mathrm{e}$ réunion régulière, tenue le 14 juillet 1976 (Résolution 78-CA-R-6).

2. Un mémoire présenté en juillet 1966 au ministre de l'Éducation par la Conférence des recteurs présente un historique détaillé des documents émanant soit d'universités, soit d'un comité des recteurs ou encore de la Conférence des recteurs, ayant trait à l'établissement d'un organisme de coordination et de planification de l'enseignement supérieur.

3. Le nom de ce comité fut modifié en 1972, lors de l'adoption du nouvel organigramme de la CREPUQ, pour devenir le Sous-comité des bibliothèques. 
coordination des bibliothèques universitaires et de voir au bon fonctionnement des projets de coopération en cours.

\section{Activités et réalisations}

Depuis sa création en 1967, le Sous-comité des bibliothèques constitue un lieu privilégié de discussion et d'échange d'informations et d'expériences en vue de coordonner et de planifier le développement des bibliothèques universitaires du Québec. Le Sous-comité joue, de plus, un rôle actif dans l'élaboration d'outils communs et dans la mise sur pied de services et de systèmes coopératifs.

En octobre 1969, à la suite d'une enquête menée auprès des bibliothèques universitaires du Québec par Jean Roy et Octave Bégin ${ }^{4}$, le comité de coordination des bibliothèques soumettait un rapport à la CREPUQ ${ }^{5}$.

Ce rapport proposait un programme d'étude et d'actions à entreprendre en vue d'une meilleure coordination des bibliothèques universitaires du Québec. Plus spécifiquement, les recommandations concernaient les collections et leur développement, leur traitement, leur mise en valeur ou leur exploitation, l'administration de l'ensemble des ressources de la bibliothèque, le budget, le personnel, les locaux et, enfin, l'automatisation des services.

Les recommandations de ce rapport furent acceptées le cinq février 1970 par le Conseil d'administration de la CREPUQ et les ressources nécessaires à la réalisation de ce programme furent mises à la disposition du comité. Celui-ci se mit immédiatement à la tâche afin d'établir les structures permettant l'organisation et le fonctionnement efficace d'un réseau coordonné de bibliothèques universitaires. Ces structures

4. Jean Roy et Octave Bégin, Enquête relative à un plan de coordination; rapport, Montréal, Conférence des recteurs et des principaux des universités du Québec, 1969.

5. Comité de coordination des bibliothèques universitaires du Québec, Rapport, Montréal, Conférence des recteurs et des principaux des universités du Québec, 1969. ont permis à chaque bibliothèque de contribuer à la richesse de l'ensemble par un apport spécifique préalablement défini en collaboration. Le but de cette collaboration demeure d'abord et avant tout - il n'est pas inutile de le rappeler - un meilleur service à la communauté universitaire.

\section{Planification et rationalisation du développement des collections}

Nous avons déjà eu l'occasion, lors du dernier congrès de I'ASTED, de décrire de façon très détaillée les efforts de coopération des bibliothèques universitaires du Québec en vue de la planification et de la rationalisation de leurs collections ${ }^{6}$.

Nous nous contenterons de signaler que l'évaluation sommaire qualitative, par sujet, du niveau actuel et du niveau de développement visé des collections des différentes institutions universitaires dont nous faisions alors mention, a été complétée au printemps 1977.

\section{Le traitement des collections}

L'augmentation substantielle du volume et du coût de la documentation, amorcée dès les années 1960 et qui n'a fait que s'accélérer depuis, a été accompagnée d'une augmentation très marquée des salaires du personnel des bibliothèques et des coûts de traitement. Très tôt, les bibliothèques ont été amenées à chercher des moyens de mise en commun de leurs ressources humaines et matérielles en vue de maintenir et d'améliorer la qualité de leurs services au public, malgré une fâcheuse tendance au plafonnement de leurs budgets.

Les premières études relatives à la centralisation et à. l'automatisation des opérations de catalogage des bibliothèques universitaires trouvent leur origine dans les recommandations du rapport Roy et Bégin.

6. Onil Dupuis, Vers une approche collective $d u$ développement des collections des bibliothèques universitaires du Québec, exposé présenté lors du $3 e$ congrès annuel de l'ASTED, Québec, 27-30 octobre 1976, 7 p. 
"Toutefois, l'initiative la plus prometteuse suscitée par ce nouvel esprit de coopération fut, sans aucun doute, le projet de création d'une centrale de catalogage pour les constituantes de I'Université du Québec, projet entrepris à l'automne 1971 sous les auspices du ministère de l'Éducation et qui devait servir de prototype aux autres institutions. Malheureusement, divers incidents de parcours - élargissement du mandat à l'ensemble des bibliothèques d'enseignement du Québec après la remise du premier rapport de juin $1972^{7,8}$; passage sous l'égide du CESIGU démantelé peu après la remise d'un second rapport en mars 19739,10, 11; retrait impromptu de l'Université du Québec de la CREPUQ quelques semaines plus tard, sans compter une opposition interne au projet venant des promoteurs d'une banque de données-maison à I'UQAM, BADADUQ (Banque de données à accès direct de I'Université du Québec) ${ }^{12}$ - firent que le projet demeura à l'état d'ébauche ${ }^{13}$."

7. Université du Québec. Commission des communications. Groupe de travail: Centrale de catalogage, Rapport intérimaire, Québec, juin 1972, 2 v.

8. Centrale de catalogage, Définition du mandat. Plan de travail, 13 novembre 1972, pag. var.

9. Centrale de catalogage, Rapport d'étape et recommandations du Groupe de travail ad hoc. Présenté aU CESIGU (Comité d'élaboration d'un système d'informatique et de gestion des universités), Québec, 26 mars 1973, 2 v.

10. Centrale de catalogage, Rapport d'étape du Groupe de travail sur une expérience-pilote avec l'Ohio College Library Center, Québec, 28 mars 1973, 5 p.

11. Centrale de catalogage, Rapport spécial du Groupe de travail sur une expérience-pilote avec l'Ontario Universities' Library Cooperative System, Québec, 13 avril 1973, 8 p.

12. Claudine Sotiau-Granger, BADADUQ, Système de repérage de l'information, Montréal, Université du Québec à Montréal, 1973, 12 p.

13. Rosario de Varennes, "Le projet conjoint de télécatalogage en coopération des universités du Québec et de I'Ontario (TELECAT/ COOP)", Documentation et bibliothèques, vol. XX, no 2 (juin 1974), 69-76.
Néanmoins, en 1973, après étude de différents systèmes d'automatisation, dont BADADUQ de I'Université du Québec, CIDBEQ du Service général des moyens d'enseignement (SGME) du ministère de l'Éducation du Québec, I'Ohio College Library Center (OCLC) et le Monograph Demonstration Project de I'Ontario Universities' Library Cooperative System (OULCS), le Sous-comité des bibliothèques recommanda ce dernier comme étant le plus apte à répondre aux besoins des universités québécoises.

UNICAT/TELECAT est un système de télécatalogage bilingue en coopération, en temps réel et en mode conversationnel. Les institutions participantes ont à leur disposition, en accès direct, les fichiers-sources ordinolingues du MARC américain et canadien (MARC/LC; MARC/CAN) et les dossiers qui correspondent aux notices du Laurentiana préparés par la Bibliothèque nationale du Québec (MARC/QUE). À partir de ces dossiers-sources, enrichis au besoin pour les rendre conformes aux normes bibliographiques communes, ou à partir des notices originales établies selon les mêmes normes, les usagers constituent un fichier collectif accessible à partir d'un point ou d'un autre du réseau.

Davantage que l'importance intrinsèque ou que la somme des énergies et des fonds consacrés au développement de ce projet, c'est sa signification au plan de la concrétisation des principes de coopération par leur application pratique à des projets d'envergure qui doit ici retenir notre attention.

UNICAT / TELECAT est un projet conjoint du Council of Ontario Universities (COU) et de la CREPUQ qui regroupe, en plus des bibliothèques universitaires québécoises et ontariennes, des bibliothèques municipales et des bibliothèques gouvernementales (provinciales et fédérales). En somme, UNICAT / TELECAT est une initiative de coopération qui dépasse les frontières trop souvent hermétiques de la régionalisation et du particularisme de chaque type de bibliothèque. 
La mise en commun des ressources de plusieurs types de bibliothèques de deux provinces a permis de réaliser, au bénéfice de chacun des participants, l'investissement considérable nécessaire au développement d'un système automatisé, applicable à l'ensemble des fonctions de la bibliothèque ${ }^{14}$ et qui constituera, en même temps, un maillon important, sinon l'embryon, d'un éventuel réseau automatisé des bibliothèques canadiennes.

\section{Mise en valeur et exploitation}

Dans le but d'assurer une exploitation plus rationnelle des ressources documentaires des différentes bibliothèques universitaires, le Sous-comité s'est d'abord préoccupé d'en faciliter l'accès.

Ainsi, le privilège d'emprunts entre bibliothèques, longtemps réservé aux professeurs et aux étudiants au niveau des études supérieures, fut octroyé, à titre expérimental d'abord, puis, en 1975, de façon permanente, à tous les étudiants de même qu'au personnel administratif de l'ensemble des établissements universitaires québécois.

Des efforts particuliers ont également été entrepris afin de réduire au minimum le délai de traitement des demandes de prêts entre bibliothèques et de faciliter les échanges entre les bibliothèques universitaires du Québec.

Dès le mois de juin 1969, le Sous-comité entreprenait les études relatives à la possibilité de la mise sur pied d'un service de messagerie reliant les bibliothèques universitaires du Québec. En décembre 1969, le service était créé et un contrat de service conclu avec l'Agence de sécurité Phillips.

PEBUQUILL (prêts entre bibliothèques des universités du Québec/Quebec Universities Inter Library Loans) est un service quotidien de messagerie reliant les bibliothèques universitaires du Québec,

14. Pierre Deslauriers, "UNICAT/TELECAT: évolution du projet et perspectives d'avenir", Documentation et bibliothèques, vol. 23 , no 3 (septembre 1977). d'une part, et, d'autre part, le réseau des bibliothèques universitaires du Québec et le réseau des bibliothèques universitaires ontariennes, le Inter University Transit System (IUTS). II met à la disposition des professeurs, des chercheurs et des étudiants de chaque université, dans un délai de 24 heures, les ressources documentaires de l'ensemble des institutions universitaires du Québec, de quinze universités de l'Ontario, de la Bibliothèque nationale du Québec ${ }^{15}$, de la Bibliothèque nationale du Canada et de l'Institut canadien de l'information scientifique et technique.

Enfin, en vertu d'une entente conclue, à titre expérimental, en 1973 et renouvelée chaque année depuis, les bibliothèques universitaires ne se facturent pas entre elles les photocopies obtenues par prêt entre bibliothèques.

Une autre entente entre les bibliothèques universitaires québécoises et ontariennes permet aux étudiants des deuxième et troisième cycles, aux professeurs et aux bibliothécaires d'emprunter directement des documents, sur présentation d'une carte émise par la CREPUQ, à l'une ou l'autre des bibliothèques universitaires du Québec et de l'Ontario.

Dans le but de faciliter la localisation des ressources disponibles, une liste des périodiques courants ${ }^{16}$ de même qu'une liste des périodiques sur microfilms ${ }^{17}$ ont

15. La Bibliothèque nationale du Québec, après avoir consenti à modifier ses règlements du prêt entre bibliothèques, fut acceptée dans le réseau PEBUQUILL le 8 février 1971.

16. Bibliothèque nationale du Québec, Périodiques canadiens sur microfilms; liste des microfilms disponibles au Québec dans les bibliothèques universitaires et à la Bibliothèque nationale; compilée par Christiane Laurin sous la direction de Jean-Pierre Chalifoux, Montréal, Ministère des Affaires culturelles, 1970 , $89 \mathrm{p}$.

17. Liste collective préliminaire des périodiques courants reçus par les bibliothèques des universités du Québec, Québec, Université Laval, 1972, $6 \mathrm{v}$.

Supplément, Québec, Université Laval, 
été compilées et publiées. De plus, la possibilité de la production automatique du catalogue des périodiques des universités du Québec et de la Bibliothèque nationale du Québec, en fonction des exigences de son utilisation pour le prêt entre bibliothèques et de la rationalisation des abonnements, est actuellement à l'étude.

Signalons en outre la publication d'un dépliant bilingue sur les services offerts à la collectivité universitaire par le réseau des bibliothèques universitaires du Québec ${ }^{18}$ et, en guise de contribution au patrimoine bibliographique québécois, une bibliographie signalétique des publications des bibliothèques universitaires du Québec et des études qui s'y rapportent ${ }^{19}$.

Enfin, un certain nombre d'outils communs de coordination et de planification ont été élaborés: protocole d'entente pour les achats de $\$ 1,000$ et plus; normes communes d'équivalence pour la cueillette de données statistiques; inventaire des collections de recherche; enquête sur les politiques de développement des collections.

\section{La gestion des ressources financières, matérielles et humaines}

II n'est pas facile, et il serait probablement fastidieux, de résumer toutes les activités de collaboration et de coopération du Sous-Comité qui se rapportent à la gestion des ressources. Un échange continu d'informations entre les membres du Sous-comité, notamment en ce qui concerne les ressources financières, constitue une part importante de ces activités qui se traduisent souvent par des interventions portant sur des questions précises auprès des autorités intéressées.

Signalons, à titre d'exemple, les pressions exercées par les membres du Sous-

18. Le réseau des bibliothèques universitaires $d u$ Québec, Montréal, Conférence des recteurs et des principaux des universités du Québec, 1976, 2 p.

19. Les bibliothèques universitaires du Québec: essai de bibliographie. Compilée par Gaétan Drolet, cette bibliographie sera publiée par la CREPUQ à l'automne 1977. comité auprès du Conseil du Trésor, lors des négociations collectives de 1976, qui ont eu pour résultat une augmentation fort substantielle des salaires des bibliothécaires.

Le Sous-comité s'est également préoccupé de favoriser, par la mise sur pied de groupes de travail ou l'organisation de rencontres, les relations entre les responsables des services de chaque bibliothèque. Ces rencontres ont pour but de permettre l'identification et la discussion d'éventuelles difficultés ou problèmes communs rencontrés dans le cadre des activités quotidiennes, l'étude de solutions possibles et la mise en commun des expériences individuelles.

Le plus important projet de coopération au niveau du partage des ressources matérielles est un projet d'entreposage en commun de la documentation rarement consultée ou la création d'un Centre universitaire bibliographique québécois (CUBIQ).

Ce projet est encore au stade des études techniques. Elles ont débuté en 1974 et portent principalement sur les points suivants:

1) prévisions relatives aux acquisitions régulières de collections ainsi que, dans certains cas, aux acquisitions spéciales de rattrapage;

2) évaluation des espaces occupés présentement par rapport aux espaces disponibles;

3) en tenant compte des données recueillies dans le cadre des études 1 et 2 , étude prospective sur les espaces dont les bibliothèques auront besoin au cours des vingtcinq prochaines années;

4) évaluation du taux de duplication des documents bibliographiques dans l'ensemble du réseau des bibliothèques;

5) enquête sur quelques centres de 
stockage américains (le Center for Research Libraries de Chicago, notamment) relativement à leurs modes de fonctionnement et de financement, au droit de propriété des institutions qui y versent des documents, etc.

Le Sous-comité étudie présentement les recommandations du rapport technique préparé à son intention par Daniel Reicher $^{20}$.

C'est à la lumière de ces études que le Sous-comité mettra la dernière main cette année au projet CUBIQ. Ce projet final devrait comporter des précisions sur l'endroit où le centre de mise en commun de la documentation rarement utilisée des universités du Québec devrait être situé, sur les espaces qui devraient y être disponibles au cours d'une période de vingt-cinq ans, sur le type de construction à envisager et son coût, sur les modalités techniques de fonctionnement et sur l'ensemble des services à offrir aux participants, ainsi que sur le budget annuel de fonctionnement.

\section{Projets et perspectives d'avenir}

Depuis l'adoption du rapport Roy et Bégin, plusieurs des objectifs mentionnés dans ce rapport ont été atteints, des projets coopératifs mis sur pied, les priorités modifiées et réajustées en fonction de nouveaux besoins; mais aucune évaluation d'ensemble, en vue de la révision et de l'élaboration de nouveaux objectifs du Sous-comité, n'a encore eu lieu.

C'est dans cette perspective que le Sous-comité des bibliothèques, lors de sa 67 e réunion tenue le 14 janvier 1977, convenait de l'opportunité d'une réflexion commune sur l'orientation et le développement des bibliothèques universitaires du Québec, le rôle du Sous-comité, l'établissement de ses priorités d'étude et d'action et l'élaboration d'un programme de travail.

Cette étude comportera trois étapes:

20. Pour une description plus détaillée, voir la "Chronique de la recherche".
1) discussion préliminaire afin d'identifier les principales tendances et les facteurs qui influenceront le développement de l'ensemble des bibliothèques universitaires ou de chacune en particulier; particulier;

2) discussion des questions identifiées en 1) au cours d'une réunion spéciale de réflexion, établissement de priorités, élaboration d'un plan de travail;

3) étude détaillée d'une question lors de chacune des réunions régulières du Sous-comité.

Une discussion préliminaire a déjà permis d'identifier les principales questions devant faire l'objet d'études et de discussions lors de cette réunion spéciale:

1) rationalisation des collections et des politiques de développement des collections;

2) politiques relatives au personnel (description de tâches, perfectionnement, etc.);

3) politique de financement;

4) politique et normes d'espace;

5) structure administrative;

6) rôle de la documentation dans le processus d'apprentissage et rôle de la bibliothèque dans la pédagogie universitaire;

7) uniformisation de la cueillette et de l'interprétation des statistiques;

8) mode de coopération des bibliothèques universitaires $\mathrm{du}$ Québec par rapport aux bibliothèques universitaires des autres provinces;

9) services à la clientèle;

10) intégration des bibliothèques 
chez

universitaires au réseau des bibliothèques québécoises;

11) intégration de la $B N Q$ au réseau des bibliothèques universitaires;

12) impact de l'automatisation sur l'allocation des ressources;

13) impact de l'utilisation de supports documentaires autres que le papier.

Cette réunion, qui eut lieu les 17 et 18 mars 1977, permit de limiter à trois les objectifs:

1) identifier les préoccupations des bibliothèques universitaires $\mathrm{du}$ Québec;

2) établir les priorités d'étude et d'action;

3) déterminer, pour chacune, les modalités précises de traitement.

Chacune des questions retenues fit l'objet d'un bref document de travail préparé par l'un des membres du Sous-comité et distribué à l'avance à tous les membres.

Au moment de la rédaction de cet article, le Sous-comité a établi, de façon préliminaire, ses priorités et un programme d'étude et d'action. II lui reste à élaborer, en fonction des objectifs identifiés pour chacun des éléments de ce programme, sa méthodologie de travail et son échéancier.

\section{Conclusion}

Les bibliothèques universitaires $\mathrm{du}$ Québec disposent des structures et des outils nécessaires à la réalisation de la rationalisation de leur développement respectif, en vue d'une plus grande accessibilité et d'une meilleure utilisation de leurs ressources. Elles ont maintenant atteint l'étape de la coopération structurée. Des projets collectifs précis en font foi.

Il leur reste à relever le défi de la planification de leur orientation.

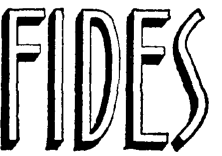

194 p. $25 \mathrm{~cm}$

LONGUEUIL ME SOURIT par

Pauline Viger-Bélanger

$\$ 5.95$

\section{LA SEIGNEURESSE} par

Robert de Roquebrune

270 p. $25 \mathrm{~cm}$

$\$ 5.95$

Deux romans de la nouvelle collection Intermondes

\section{LA SEIGNEURIE \\ DE}

PHILIPPE AUBERT DE GASPE

Saint-Jean-Port-Joli

par

Jacques Castonguay

dans la collection Loisirs et culture

162 p. $19.5 \times 19.5 \mathrm{~cm}$

$\$ 6.95$

\section{LE RU D'IKOUE \\ par \\ Yves Thériault}

dans la collection du Goéland

127 p. $20.5 \mathrm{~cm}$

$\$ 4.95$.

VERS UN RENOUVEAU DE LA

THÉOLOGIE SACRAMENTAIRE par

Raymond Vaillancourt

dans la collection Liturgie vivante

164 p. $19 \mathrm{~cm}$

$\$ 5.00$

HISTOIRE DE LA PROVINCE DE QUEBEC

Tome IX: Marchand par

Robert Rumilly

316 p. $19.5 \mathrm{~cm}$

$\$ 8.00$

SEXUALITE ET FOI

par

Guy Durand

dans la collection Héritage et Projet, 19

426 p. $21.5 \mathrm{~cm}$

$\$ 10.00$

En vente dans toutes les librairies et à la

LIBRAIRIE GENERALE FIDES

235 est, Dorchester, Montréal 861-9621 